\title{
Moving object detection and shadow removal algorithm
}

\author{
Wang Mengqiao Yang Jie \\ Key Laboratory of Fiber Optic Sensing Technology and Information Processing, \\ Ministry of Education, Wuhan University of Technology, Wuhan 430070 \\ e-mail:2457254782@qq.comｅ-mail:466802324@qq.com
}

Keywords: target detection; shadow removal; Gaussian background model; RGB color information Abstract:To solve problem of the moving object's detection and shadow removal with a fixed camera,firstly we use the traditional method-Gaussian Background Model-to extract the background and detect the target with background subtraction algorithm, then we use RGB color information to detect shaded parts in foreground area based on RGB color model by building Gaussian mixture shadow model algorithm to eliminate shadows, finally the experiments have verified the algorithm of this paper,proved that the algorithm can detect the shadow of the target simply,and it can also remove the shadow effectively.

\section{Introduction}

With technology's fast development and people's growing demand, the video surveillance technology is also developing fast and is being widely applied in many fields, like intelligent surveillance,transportation and military. Moving target's detection and shadow removal are not only important in computer vision processing, but also the core of intelligent transportation systems, the development of its technology and the prospects is concerned by many people.

Moving target's detection is to extract the foreground out from image sequences which has relative motion with background, and to further divide motion outlook into independent target by two-dimensional image features,such as gray scale, edge and texture ${ }^{[1]}$.Background subtraction based on gaussian mixture model in this paper is adopted to detect the target, which is more effective than the simple background subtraction.

Sunshine and lighting may cause shadow in foreground image, mutual occlusion between scenes or uneven illumination may cause shadow in the imaging process. The shadow will directly affect the target's track and detection. It damages to precision in image matching, accuracy in pattern recognition and automation in target extraction. Therefore, shadow removal is very important.The research about image's shadow processing began in early 1990s, including the shadow detection and shadow removal.The research about shadow detection and removal algorithm have developed nearly 20 years, and have achieved certain results, they are widely applied in traffic surveillance, face recognition, image segmentation and other fields.Shadow detection algorithm based on RGB color model is proposed in this paper. The article also validates the algorithm according to experiments to determine that the algorithm can detect and remove the moving target's shadow better, and it's easy to implement.

\section{Gaussian mixture model}

\section{1)Background subtraction}

Currently the main three methods of the detection and extraction of the moving targets are 
frame difference method, background subtraction and optical flow. Background subtraction ${ }^{[2]}$ is a method to use of the current frame and background model difference operation, and by setting a certain threshold, the difference image is binarized to detect the moving goals. Background subtraction's thoughts is similar to frame difference's, other forms are compared common points and different points with template. Background difference is based on this thinking, and background image overlapped portion will be defaulted to the background, different sections will be extracted as a foreground image.

Let $f(x, y)$ is an image sequence, the current frame is denoted as $f_{k}(x, y)$, the current background image is expressed as $b_{k}(x, y)$, the differential image can be expressed as:

$$
d_{k}(x, y)=\left|b_{k}(x, y)-f_{k}(x, y)\right|
$$

Then select the appropriate threshold for the differential image processing binarization, binarization image is expressed as:

$$
\mathrm{r}_{\mathrm{k}}(x, y)=\left\{\begin{array}{l}
1, d_{k}(x, y) \geq T \\
0, d_{k}(x, y)<T
\end{array}\right.
$$

Where, $r_{k}(x, y)$ is the image after binarization, $T$ is a preset threshold.

The method of background subtraction just needs a small amount of computation but extract more complete moving target, so as to realize the detection of target.The key of the algorithm is to select background model, if the background model is not updated, when the light changes or background disturbance accumulation exceeds a set threshold in time, it will bring the background mistaken for a moving target, resulting in the error detection result, so to establish an effective background model is the core of background subtraction method.

\section{2) Gaussian mixture model}

Gaussian mixture model ${ }^{[3]}$ is a background model composed of multimodal Gaussian distribution,it combines a number of single Gaussian model to express complex scene corresponding pixel value, namely each pixel value of an image are composed of a plurality of Gaussian distribution. Formula (2-3) shows the relationship between Gaussian mixture model and a single Gaussian model:

$$
\mathrm{f}\left(\mathrm{X}_{\mathrm{t}}=x\right)=\sum_{\mathrm{i}=1}^{\mathrm{k}} w_{i, t} N\left(x, u_{i, t}, \sum_{i, t}\right)
$$

Where, $K$ is a predetermined value, which determines the accuracy of calculation , $w_{i, t}$ indicates a weight value. According to the formula, Gaussian mixture model is a series of single weights and Gaussian distribution model, in which:

$$
N\left(x, u_{i, t}, \sum_{i, t}\right)=\frac{1}{\sqrt{2 \pi \mid \Sigma}} \exp \left[-\frac{1}{2}\left(x-u_{i, t}\right)^{t} \sum_{i, t}^{-1}\left(x-u_{i, t}\right)\right]
$$

Where, $u_{i, t}$ represents the model expectations, $\Sigma_{i, t}$ is the variance of the model, This paper is using sample mean that get by sampling to determine the value of $u_{i, t}$ and determine the value of $\Sigma_{i, t}$ with the sample variance.

In order to adapt to the slow change of light better, Gaussian mixture model is compared the value of the current pixel point with the center of the Gauss distribution, and by selecting a certain weight coefficient to update the current center position.

Algorithm procedure is as follows:

(1) Model Initialization. Each pixel value to be detected is matched with the current model of the K corresponding to the Gaussian distribution, if the matching pattern is consistent with the background required, the corresponding pixel belongs to the background pixels, otherwise it is 
foreground pixels.

(2) Parameter update. Each mode weights according to equation (2-5) update. Where, a represents a learning rate, corresponding to a pattern that matches $\mathrm{M}_{\mathrm{k}, \mathrm{t}-1}=1$, otherwise $\mathrm{M}_{\mathrm{k}, \mathrm{t}-1}=0$.

$$
w_{k, t}=(1-\partial) w_{k, t-1}+\partial\left(M_{k, t}\right)
$$

(3) Matching arrangement. If there is no pattern matched with (1), then the smallest model weights will be replaced, the average value of the model will be the current pixel value, standard deviation of the model will be an initial larger value, the weight of the model will be a smaller value.

(4) Get the background. Select the first B model as a backdrop, B can be expressed as (2-6) as follows:

$$
B=\arg \min _{b}\left(\sum_{k=1}^{b} w_{k}>T\right)
$$

Gaussian mixture model background is a Gaussian distribution, when the prospect data aggregation reaches a certain level, the still people can be seen as a new Gaussian distribution, it means the stop person has become a backdrop, but once people is moving even it is slow motion will be difficult to form a Gaussian distribution. Thus, the above shows that Gaussian mixture model especially suitable for the detection of slow-moving objects.

\section{Shadow removal algorithm based on RGB space}

Due to the influence of light and other factors,the resulting of foreground image contains shaded areas, The detection of moving objects will be affected. In light of this, we use a RGB color information to detect foreground area shaded so that we can detect the real moving object .

\section{1)RGB shading model}

It observed that shadow just change the current pixel brightness, so the impact of a shadow on RGB space to pixel are mainly the following two aspects:

(1) When the current pixel point has a shadow, the value of the RGB components of its pixels is smaller than without a shadow;

(2) When the current pixel point has a shadow, the reduction of pixel value on its RGB components is same. Thus, the shadow model based on the RGB space can be obtained.

$$
\left\{\begin{array}{l}
S_{r}<\mathrm{B}_{\mathrm{r}}, \mathrm{S}_{\mathrm{g}}<\mathrm{B}_{\mathrm{g}}, \mathrm{S}_{\mathrm{b}}<\mathrm{B}_{\mathrm{b}} \\
\mathrm{S}_{\mathrm{r}:}: \mathrm{S}_{\mathrm{g}}: \mathrm{S}_{\mathrm{b}}=\mathrm{B}_{\mathrm{r}}: \mathrm{B}_{\mathrm{g}}: \mathrm{B}_{\mathrm{b}}
\end{array}\right.
$$

Where: $B_{r}, B_{g}, B_{b}$ are the value for the three components of the RGB of the current background pixel; $S_{\mathrm{r}}, S_{\mathrm{g}}, \mathrm{S}_{\mathrm{b}}$ are the value of the current image sequence detection of RGB pixels.

\section{2) The design of RGB shadow detection algorithm}

The algorithm uses the seed filling ideas to deal with the shaded area which has connectivity problems.From the article's analysis above, we can know that the fluctuations of B color is larger,So it had not been carried out to detect shadows. The steps of this algorithm are described as follow:

(1) Input the original color image and give judging conditions of shaded areas and non-shaded areas :

$$
f(R)<70 \| f(G)<70 \& \& T>25
$$

(2) Do convolution operation to $\mathrm{R}$ and $\mathrm{G}$ components of color image by using gauss Laplace operator.If there are pixels satisfy the judging conditions of step (1), then let the pixel $R=255, G=$ $255, \mathrm{~B}=0$;

(3) Repeat the Step (2) until the calculation is converges, then get the results of shadow test;

(4) When the result of shadow detection is unsatisfactory,we can change the threshold value, then repeat steps (2) and (3) until you get a satisfactory detection. 


\section{3) The design of RGB Shadow Removal Algorithm}

Based on the analysis above,we can get the shadow removal algorithm as follows:

(1)Establish Gaussian mixture model and make matching operation to entered pixels, If the match is successful, then the pixel is a background pixel;

(2)Update foreground pixels;

(3)Establish gaussian mixture shadow model and make matching operation.If successful,then the pixel is a shadow pixel,otherwise, it is foreground moving pixels;

(4)Update movement pixels.

\section{Experimental results and analysis}

In this paper,under Window7 operating system and VS2010 environment, using OpenCV2.4.10 for software development.In order to achieve satisfactory results, Gaussian distribution numbers are both set to 3 when modeling.In the experiment,I use the method of the binary processing to carry out the prospects diagram,the threshold is set to 30.The final results is shown in Fig. 1.

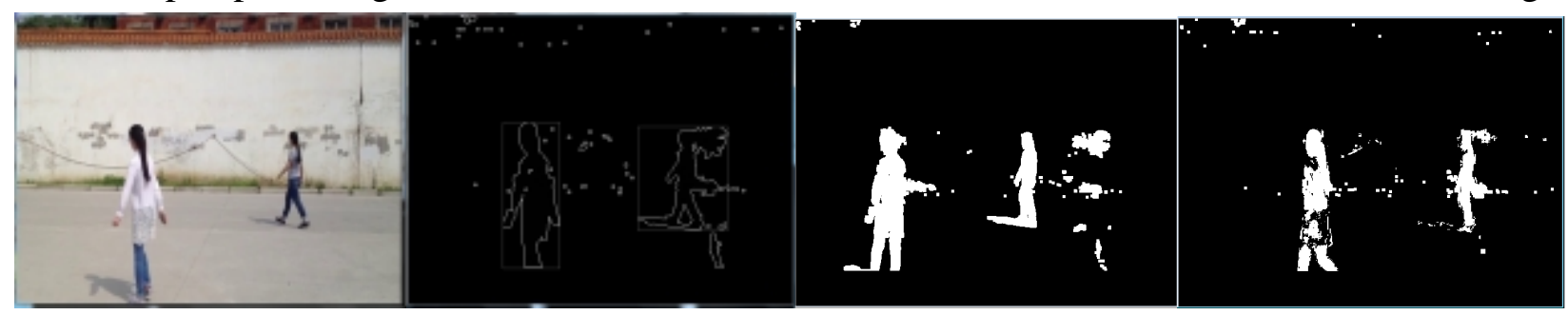

(a)original picture

(b)prospects picture

(c) background subtraction

(d)shadow removal

Fig. 1 the results of this experiment

Shadow removal algorithm based on RGB can ensure the removal of the shadow of moving objects without distortion contour,It can suppress noise significantly, and can achieve the speed that real-time detection required.

\section{Conclusions}

In this paper, while we use the traditional Gaussian mixture model for modeling background, it can achieve a good extraction of moving objects. But there will be "holes" phenomenon in the foreground area to slow-moving target with the same color. According to the characteristics of the shadow pixels in the RGB color space,matching the shadow model with the current pixel RGB space,then establish Gaussian mixture shadow model corresponded to the current outlook by using Gaussian mixture model, the shadow pixels can be removed to meet the current Gaussian mixture shadow model and detect foreground object better. This shadow removal method works well, can be applied to common scenarios shadow and with high real-time .The results of this research can be used in many areas such as monitor systems.

\section{Acknowledgements}

This work was supported by National Science Foundation of China (NSFC) under Grant 51479159.

\section{References}

[1]Ning Wang. Shadow detection and removal algorithm for image[D]. Beijing Jiaotong University, 2011, In Chinese. 
[2]Zefeng Li, Lingyun Zhu. Moving target detection method based on improving background subtraction method[J] .Instrumentation Technology, 2012,In Chinese.

[3]Jing Liu, Ling Wang. A Gaussian mixture model background improved algorithm[J]. Computer Engineering and Applications .2010,In Chinese.

[4]Yan Wei. target detection and blanking research and application based on background update[D]. Anhui University, 2013,In Chinese.

[5]Yan Wei,Zhengzheng Tu,Aihua Zheng, Bin Luo. Vanishing algorithm combinated RGB color and texture features[J]. Computer Technology and Development, 2013,In Chinese.

[6]Yanhong Wang. Moving target detection and tracking algorithm study based on OpenCV[D]. Hangzhou University of Electronic Science and Technology, 2013, In Chinese.

[7]Saunier N,Sayed T.A feature-based tracking algorithm for vehiclesinintersections[C].Proceedings of the 3rd Canadian Conference on Computer and Robot Vision（CRV'06）,2011. 